\title{
2020. Año Internacional de las Enfermeras y las Matronas
}

\author{
Rodolfo Crespo-Montero \\ Hospital Universitario Reina Sofía de Córdoba. Córdoba. España
}

\begin{abstract}
Como citar este artículo:
Crespo-Montero R. 2020. Año Internacional de las Enfermeras y las Matronas.

Enferm Nefrol. 2020 Ene-Mar;23(1):7-8
\end{abstract}

La Organización Mundial de la Salud (OMS) ha declarado 2020 como el Año Internacional de las Enfermeras y Matronas. A esta iniciativa se han unido la Confederación Internacional de Matronas, el Consejo Internacional de Enfermeras (CIE), la campaña Nursing Now y el Fondo de Población de las Naciones Unidas (UNFPA), y durará un año entero.

Para la OMS, las enfermeras y matronas desempeñan "una función crucial en la prestación de servicios de salud, ya que consagran sus vidas a cuidar a las madres y los niños, administrar vacunas que salvan vidas, proporcionar consejos de salud, cuidar de las personas mayores y, en general, satisfacer las necesidades sanitarias esenciales del día a día. Además, suelen ser el primer y el único lugar de atención disponible en sus comunidades. A fin de lograr la cobertura sanitaria universal, el mundo necesita que el personal de enfermería y partería aumente en 9 millones ${ }^{\prime \prime}$.

Este organismo internacional ha preparado una serie de mensajes institucionales y material para hacer más visible el tema elegido. Dentro de los Mensajes institucionales que dedica al personal de Enfermería y Partería, destaca Cinco ámbitos de inversión fundamentales²:

- Invertir en servicios dirigidos en mayor medida por el personal de enfermería y el de partería, que de este modo puede trabajar dando lo mejor de sí mismo.

- Emplear a personal de enfermería más especializado.

- Otorgar al personal de enfermería y partería un lugar central en la atención primaria de salud, desde el que asuma la prestación de servicios y la supervisión de los agentes de salud comunitarios.

- Respaldar al personal de enfermería y partería en las labores de promoción de la salud y prevención de enfermedades.

- Invertir en liderazgo de los servicios de enfermería y partería.
Esta campaña de visibilización de las profesiones enfermera y matrona es muy importante, pues supone una llamada de atención a las autoridades sanitaria de los distintos países sobre el rol que estas profesiones pueden y deben desempeñar en la asistencia sanitaria mundial, y sobre todo, por la repercusión mediática que las iniciativas de la OMS tienen a nivel global. Sin embargo, y curiosamente en un país con un gran Sistema Nacional de Salud, en España estamos lejos de conseguir el protagonismo profesional que tanto la OMS como el CIE reclaman para nuestra profesión.

Como primer problema sin resolver, y para mí más importante, seguimos siendo uno de los países pertenecientes a la Organización para la Cooperación y el Desarrollo Económico (OCDE) con las ratio enfermera/ paciente más baja en comparación con el resto de países. Este problema se ha convertido en crónico en nuestra sanidad y se ve agravado por diferencias internas entre las distintas Comunidades Autónomas (CCAA) que conforman el estado español. Según los datos que ha publicado la OCDE en su informe "Health Statistics 2019", tanto en la ratio de Enfermería como en el de Medicina se ha mejorado algo el último año analizado, aunque no terminan de destacar respecto a los demás países. Dicho informe señala que la media de la OCDE en lo que a ratio de Enfermería se refiere se encuentra en 8,8 enfermeras por cada 1.000 pacientes. Una cifra que está tres puntos por encima de la media española, situada en 5,7 enfermeras por cada 1.000 habitantes, ocupando el puesto 28 de 40, de una clasificación que lidera Noruega, con un ratio de 17,6 enfermeras por cada 1.000 pacientes $^{3}$.

Otro problema importante sin resolver es el desarrollo e implantación real de las Especialidades de Enfermería. Después de 14 años, que no esté desarrollado al completo el RD de Especialidades de Enfermería, no sólo es un 
despropósito, sino que raya en la malversación de fondos públicos, porque en pleno 2020 que sólo esté implantada la Especialidad Obstétrico-Ginecológica (Matrona), es de una falta de respeto por parte de las administraciones sanitarias de las diferentes CCAA, que no merece siquiera calificativo. Esto en cuanto a Especialidades que tienen su Plan Formativo desarrollado y existen bolsas de especialistas en todo el país, porque de la Especialidad de Cuidados Médico-Quirúrgicos ni se tiene Plan Formativo ni se les espera.

A esta resistencia pasiva para que se implanten las Especialidades de Enfermería en todas las CCAA hay que sumar el escaso interés mostrado por las autoridades sanitarias en el desarrollo de otro rol profesional que podría suplir la falta de especialistas en algunas áreas asistenciales muy concretas, como es la Enfermería de Práctica Avanzada, bien por la vía de Acreditación de Competencias Específicas de Intervención Avanzada, modelo desarrollado en Andalucía por la Agencia de la Calidad Sanitaria Andaluza ${ }^{4}$, o bien por la vía de la Ley de Ordenación de las Profesiones Sanitarias, con los Diplomas de Acreditación y los Diplomas de Acreditación Avanzada $^{5}$. En definitiva, existe una inercia negativa que condiciona esta "resistencia pasiva", que hace imposible cualquier atisbo de empoderamiento real de la enfermería: ocupar puestos de responsabilidad en cargos estratégicos en la toma de decisiones políticas, solución real a los especialistas de enfermería, en los que el erario público ha gastado presupuesto en su formación, reconocer que actualmente la asistencia sanitaria es tan amplía y superespecializada, que para determinados puestos de trabajo ya no vale la "enfermera para todo", que hay que desarrollar el modelo de los Diplomas de Acreditación y los Diplomas de Acreditación Avanzada, que colmaría también las expectativas profesionales de diferentes colectivos, como por ejemplo en la Enfermería Nefrológica.

En fin, cualquier paso adelante hacía una mayor profesionalización de la enfermería, y por ende, mayor protagonismo en el proceso asistencial, tiene en contra a esa fuerza "invisible", que es quien toma decisiones y rige el destino profesional de la profesión enfermera.

Todo lo demás, como las iniciativas de la OMS y el CIE están muy bien porque recuerdan el papel que debería ejercer enfermería en los diferentes modelos de atención sanitaria, pero no dejan de ser meras declaraciones de intenciones, muy alejadas del día a día del ejercicio profesional de enfermería, que en ocasiones quedan tan sólo en eslóganes que uno lleva oyendo muchos años, y que eso si, ahora tienen más resonancia porque se difunden por redes sociales, pero con el mismo resultado de siempre.

\section{Referencias}

1. Organización Mundial de la Salud. [Consultado 06 feb 2020]. Disponible en: https://www.who.int/es/ news-room/campaigns/year-of-the-nurse-and-themidwife-2020.

2. Organización Mundial de la Salud. Mensajes fundamentales. [Consultado 06 feb 2020]. Disponible en:https://www.who.int/es/news-room/campaigns/ year-of-the-nurse-and-the-midwife-2020/get-involved/key-message.

3. Redacción Médica. OCDE: España escala al puesto 11 en número de médicos y al 28 en enfermeros. [Consultado 06 feb 2020]. Disponible en: https:// www.redaccionmedica.com/secciones/sanidad-hoy/ ocde-espana-escala-al-puesto-11-en-numero-de-medicos-y-al-28-en-enfermeros-8984.

4. Agencia de Calidad Sanitaria de Andalucía. Consejería de Salud y Familias. Junta de Andalucía. [Consultado 06 feb 2020]. Disponible en: https://www. sspa.juntadeandalucia.es/agenciadecalidadsanitaria/ acsa_profesionales/registro.rutas.asp?modo=PB.

5. Real Decreto 639/2015, de 10 de julio, por el que se regulan los Diplomas de Acreditación y los Diplomas de Acreditación Avanzada.

Este artículo se distribuye bajo una Licencia Creative Commons Atribución-NoComercial 4.0 Internacional. https://creativecommons.org/licenses/by-nc/4.0/

Open Access (c) (1) () 